\title{
Toxicity of advanced glycation end products (Review)
}

\author{
ALEKSANDRA KUZAN \\ Department of Medical Biochemistry, Faculty of Medicine, Wrocław Medical University, Wrocław 50-368, Poland
}

Received November 12, 2020; Accepted January 26, 2021

DOI: 10.3892/br.2021.1422

\begin{abstract}
Advanced glycation end-products (AGEs) are proteins or lipids glycated nonenzymatically by glucose, or other reducing sugars and their derivatives, such as glyceraldehyde, glycolaldehyde, methyloglyoxal and acetaldehyde. There are three different means of AGE formation: i) Maillard reactions, the polyol pathway and lipid peroxidation. AGEs participate in the pathological mechanisms underlying the development of several diseases, such as diabetes and its complications, retinopathy or neuropathy, neurological disorders (for example, Parkinson's disease and Alzheimer's disease), atherosclerosis, hypertension and several types of cancer. AGE levels are increased in patients with hyperglycaemia, and is likely the result of the high concentration of glycation substrates circulating in the blood. The present review summarises the formation and nomenclature of advanced glycation end-products, with an emphasis on the role of AGEs in the development of diabetes, neurological disorders, as well as in cancer and other pathologies. A particular focus is placed on the functions of toxic AGEs. Additionally, studies which have shown the cytotoxicity of glycated albumin and other AGEs are also discussed. Finally, the diagnostic relevance of AGEs as well as for targeting in therapeutic strategies are highlighted.
\end{abstract}

\section{Contents}

1. Introduction

2. Cytotoxicity of AGE

3. Methylglyoxal (MGO)-AGE

4. TAGEs in diabetes

5. TAGEs in the nervous system

6. TAGEs in cancer

7. Dietary AGEs (dAGEs)

8. TAGEs for diagnosis and treatment of diseases

9. Methodology for determining AGEs in biological materials 10. Summary and conclusions

Correspondence to: Dr Aleksandra Kuzan, Department of Medical Biochemistry, Faculty of Medicine, Wrocław Medical University, ul. Chałubińskiego 10, Wrocław 50-368, Poland

E-mail: aleksandra.kuzan@umed.wroc.pl

Key words: glycation, toxicity, diabetes, cancer, neuropathy, advanced glycation end products

\section{Introduction}

Advanced glycation end-products (AGEs) represent a broad heterogeneous group of compounds formed by nonenzymatic reactions between reducing sugars or oxidized lipids and the free amino groups of proteins, amino phospholipids or nucleic acids. There are three different methods of AGE formation, which are schematically depicted in Fig. 1 (1-4).

The initial process, known as the Maillard reaction, leads to the formation of glycated molecules termed Amadori products or early glycation products. Further rearrangement, oxidation, reduction, dehydration, condensation, fragmentation and cyclization of an Amadori product results in the formation of relevant irreversible AGEs. Incubation of proteins with lipid peroxidation products is an alternative method of generating AGEs. The polyol pathway leads to the conversion of glucose into fructose, and also promotes glycation; fructose may further be converted to 3-deoxyglucose and fructose-3-phosphate, both of which are very potent nonenzymatic glycation agents (5-7). Detailed information on AGE formation is reviewed elsewhere (8-11).

In studies on AGE, two classifications for the nomenclature regarding AGEs are used, which are either focused on the structure of the AGE or instead focused on the proteins being modified. In the first classification system, the most extensively studied AGEs are $N$-carboxymethyllysine (CML), pentosidine, crossline, pyrraline and hydroimidazolone (2). The second group includes AGE-1 (glucose-derived AGEs), AGE-2 (glyceraldehyde-derived AGEs), AGE-3 (glycolaldehyde-derived AGEs), AGE-4 (MGO/methylglyoxal-derived AGEs), AGE-5 (glyoxal-derived AGEs), AGE-6 (3-deoxyglucosone-derived AGEs) and acetaldehyde-derived AGEs (AA-AGEs) $(12,13)$. Specific modifications of proteins are also considered AGEs; for example, haemoglobin (also referred to as HbAlc, is in fact an Amadori product, not an AGE), albumin, eye crystallin, collagen type IV and others (Fig. 2) (1-3,14).

It has been reported that AGEs adversely affect several processes by two primary mechanisms: Directly through trapping and cross-linking of proteins, and indirectly by binding to specific receptors for AGE on the surface of various cells. Upon binding to receptors, AGEs activate several signalling pathways, including NF- $\kappa B$, MAPKs, and Jun N-terminal kinase, which regulate the transcription of proteins, such as cytokines, chemokines, growth factors, adhesion molecules and extracellular matrix proteins. In doing so, AGEs can result alter chemotaxis, angiogenesis, oxidative stress, cell proliferation and programmed cell death $(5,7)$. 
AGE molecules (free, peptide-bound and protein-bound) are found in the blood plasma in high concentrations, particularly in diabetic patients. This is explained by their high concentration of the substrates; glucose and its derivatives in blood (5). AGEs are also found in aging patients and those with degenerative diseases, where AGEs accumulate in cells and tissues, such as arteries, neurons and hepatocytes (6).

Glycation may also occur whilst cooking foods, for example during frying, baking, heating food in a microwave oven, and especially during caramelization. The resulting products have a strong taste and aroma. Another source of exogenous AGEs is cigarette smoke (15). Some of the exogenous AGEs are carcinogenic, for example acrylamide or heterocyclic amines, whereas others interfere with cell signal transduction or expression of numerous genes (16-18). Both endogenous and exogenous AGEs bind to specific receptors, and cause oxidative stress and promote inflammatory processes $(16,19)$.

It is postulated that AGEs participate in the pathological mechanism of cardiovascular disease, nephropathy, rheumatoid arthritis, dysfunctions in bone remodelling and neurological diseases (for example, Alzheimer's and Parkinson's disease), cancer growth, metastasis and other degenerative diseases $(9,20-22)$. The list of pathological conditions associated with AGE with a short description of the relationship between glycation and the disease is presented in Table I.

AGEs may also be considered glycotoxins, due to their toxic effects on certain cells and tissues (5). The term 'TAGE' is the abbreviation for toxic advanced glycation end-products, which applies to some (AGE-2, AGE-3 and AA-AGE), but not all AGEs. These molecules induce reactions primarily by interaction with receptors for advanced glycation end-products (RAGEs), and exert their toxic effects in the blood vessels, liver and retinas, and can also promote the development of several types of cancer as well as infertility $(12,13,21-23)$. There is considerable research showing that non-TAGE molecules, such as CML, pentosidine, pyrraline and crossline may also be cytotoxic (24-26).

\section{Cytotoxicity of AGEs}

The viability of various types of cells in the presence of AGEs has been extensively studied. In all reported studies, changes in the survival rate of cells in the presence of most types of AGEs was demonstrated. Below, a brief overview of the studies examining the effects of AGEs on viability of cells is discussed.

The toxicity of CML (the best-defined AGE) was investigated in mice. The estimated $\mathrm{LD}_{50}$ of CML was $>5 \mathrm{mg} / \mathrm{kg}$. Administration of 2 or $5 \mathrm{~g} / \mathrm{kg} \mathrm{CML} \mathrm{did} \mathrm{not} \mathrm{induce} \mathrm{mortality}$ within 14 days. However, some biochemical and histopathological changes were observed: Markers of aberrant hepatic and renal function were elevated, antioxidant enzyme (SOD and GSH-Px) activities were reduced, the levels of a marker of lipid peroxidation (MDA) were increased and histological changes were observed in the lungs, liver, kidney and spleen (24).

The effect of glycated albumin has been investigated in numerous different cell lines. For example, the impact of BSA-AGE (BSA incubated for 12 weeks with glucose) on cell cultures of amniotic and embryonic origin (WISH and MRC-5 cell lines) and on placental villi explants were determined. The observed effects included: Condensation of chromatin, formation of apoptotic bodies and elevated expression of cytokeratin 18 and Caspase 3. It was concluded that BSA-AGE had a direct toxic effect on the cell viability in a time and dose-dependent manner, and that some pregnancy complications may arise due to the formation of AGEs (5).

The effect of glycated albumin was also analysed on other mammalian cell lines: Peripheral blood mononuclear cells, 293 cells, normal human fibroblasts and Chinese hamster ovary cells. Glycated albumin was significantly more toxic than native human serum albumin $\left(\mathrm{LC}_{50}\right.$ values between $35.00-48.34$ vs. $5.47-9.10 \mu \mathrm{g} / \mathrm{ml}$, respectively) (2). The addition of rosmarinic acid suppressed the cytotoxic effects and inhibited the activity of elastase and collagenase (2).

The cytotoxicity of BSA-AGE was investigated in BHK 21 hamster fibroblast cells and SH-SY5Y human neuroblastoma cells. It was found that BSA-AGE significantly induced cell death in a dose-dependent manner, which was confirmed by three different methods (Thiazole Blue assay, lactate dehydrogenase assay, Neutral Red assay). Notably, the cytotoxic effects of AGEs were attenuated by antioxidants, such as thioctic acid and $\mathrm{N}$-acetylcysteine, which lead to the conclusion that the toxic effects of AGEs was associated with the oxidative stress (3). Chowdhury et al (27) drew similar conclusions in a study performed using D-galactose-derived AGEs. The experiments were performed using a mouse model; galactose was injected and one group of mice were administered antioxidants, which abolished the toxic effects (27). Similar results were observed when assessing the effects of AGEs generated from ribose. In a study conducted on Chinese hamster ovary cells, the effect was suppressed by glucose-regulated protein $78 \mathrm{kDa}$ (GRP78). Glycation with a ribose is called ribosylation (6). GRP78 acts as a chaperone, and when it is ribosylated, which induces ER stress, it leads to cell death (6).

\section{Methylglyoxal (MGO)-AGE}

MGO is a by-product of glycolysis. This highly reactive dicarbonyl compound is also a major precursor of AGE-4, a methylglyoxal-derived AGE. MGO is not classified as a TAGE; however, its cytotoxicity has been repeatedly observed.

Sampath et al (28) showed the cytotoxic effects of MGO on Human Retinal Pigment Epithelial cells. It was found that MGO-induced cytotoxicity resulted in increased levels of AGEs, such as CML, as well as expression of various RAGEs and glutathione. In the presence of AGE-MGO, the translocation of Nrf2 from the cytosol to the nucleus is inhibited, which results in decreased expression of detoxifying enzymes such as heme oxygenase-1 (28). It is also worth quoting the results of research on the impact of MGOs on bone marrow-derived endothelial progenitor cells (EPC). MGO increased the levels of AGEs, and decreased cell viability and protein expression of vascular endothelial growth factor receptor (VEGFR)-2. The latter is associated with functional impairments of tube formation. Inhibition of RAGEs by FPS-ZM1 significantly reverses the decrease in VEGFR-2 protein expression and angiogenic dysfunction in MGO-treated EPC (29). 


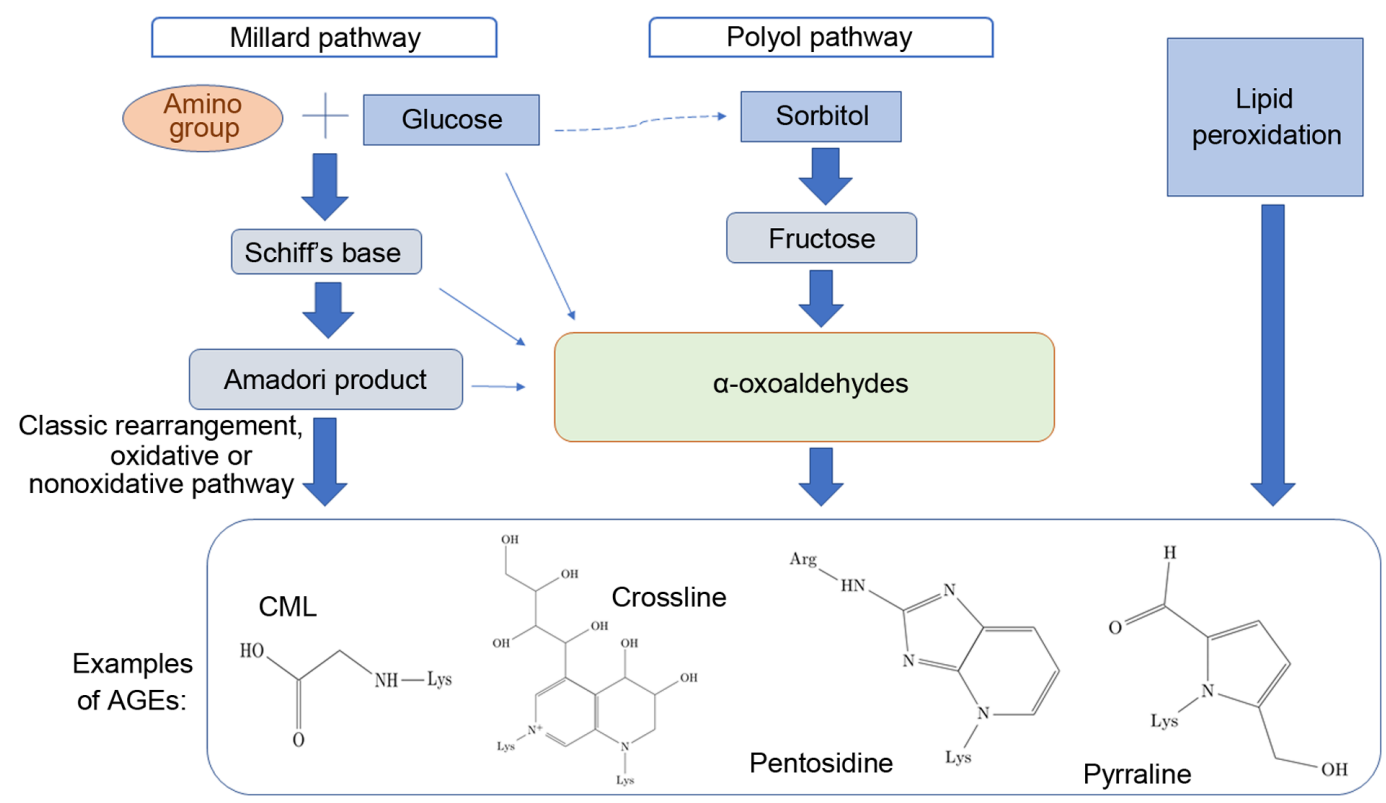

Figure 1. AGE formation pathways, including the Millard pathway, polyol pathway and lipid peroxidation pathway. The structural formulas of the most common types of AGEs are also shown, based on Khan et al (9). AGE, advanced glycation end product; CML, carboxymethyllysine.

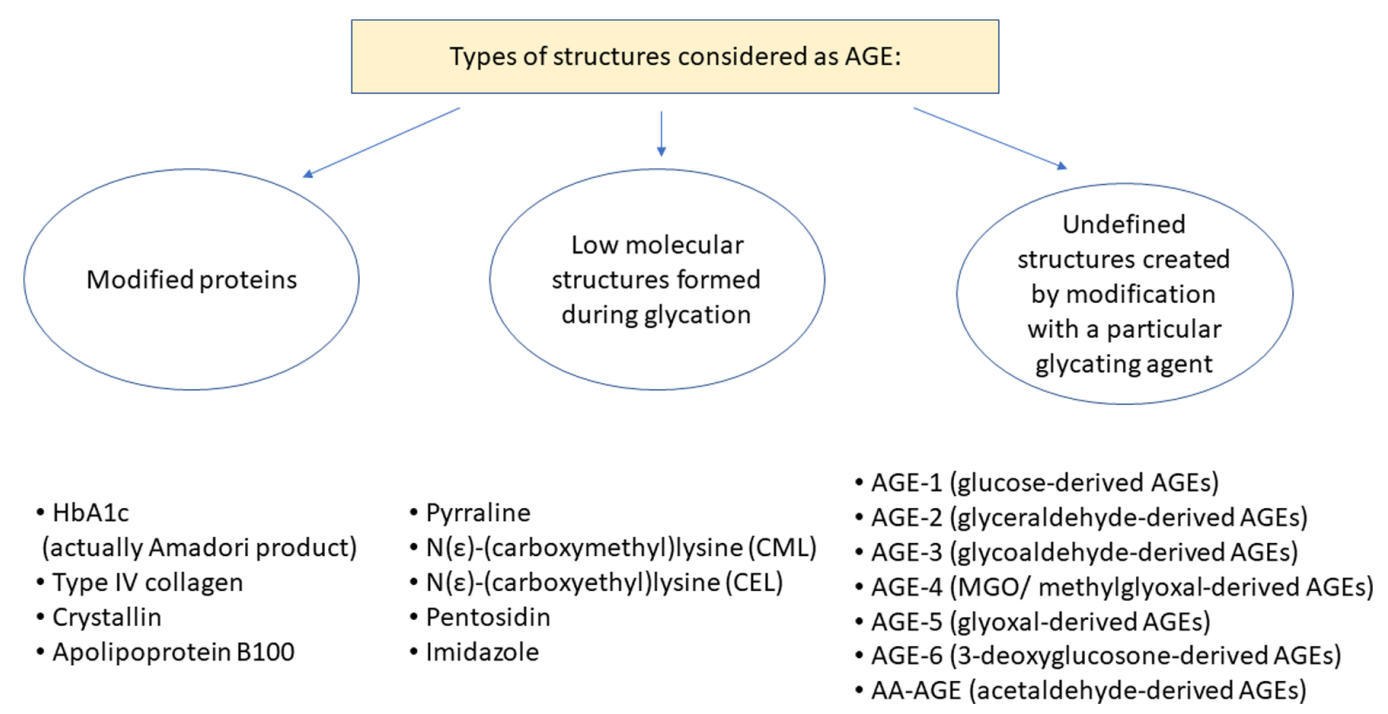

Figure 2. Classification of AGEs based on the type of structure and the origin of the substrates. AGE, advanced glycation end product.

\section{TAGEs in diabetes}

TAGEs are a subset of AGEs, which include AGE-2, AGE-3 and i AA-AGE. Diabetic hyperglycaemia may be caused by elevated production of TAGEs. Histological analysis of healthy control rats and STZ-induced diabetic rats showed AGE-2 expression in the brain, pancreas and stomach, but not in the adipose tissue, intestine, eye, heart, kidneys, spleen or lungs. However, there seemed to be no difference in the distribution of AGEs between the control group and experimental group (1).

As a common complication of diabetes is retinopathy, it is not surprising to find reports showing the involvement of AGEs in the death of retinal tissue. Takeuchi and Yamagishi (12) reported that in the presence of TAGEs, apoptotic cell death of retinal pericytes was observed, and this was mediated by
TAGE interaction with RAGEs. It was found that AGE-2 and AGE-3 accelerated retinopathy by upregulating vascular endothelial growth factor (VEGF) mRNA levels, as well as stimulating DNA synthesis and tube formation in microvascular endothelial cells via the interactions with RAGEs (12).

Another common complication of diabetes is nephropathy. It has been demonstrated that TAGEs influence nephropathy via two mechanisms: i) They induce apoptotic cell death in human mesangial cells; and ii) cause hyperfiltration and microalbuminuria by stimulating secretion of VEGF and monocyte chemoattractant protein-1 (12).

\section{TAGEs in the nervous system}

Of all the TAGEs, AGE-2 and AA-AGE appear to be particularly neurotoxic. It was demonstrated that AGE-2 exhibits 
Table I. Effects of AGEs in various diseases.

Types of AGEs

Disease/condition

Diabetic retinopathy

implicated in disease

AGE-2, AGE-3
Role of AGEs in disease

(Refs.)
TAGE

Diabetic nephropathy

Diabetic neuropathy

Other neuropathies

(including Parkinson's

disease)

Malignant melanoma

Other types of cancer

\begin{abstract}
Alzheimer's disease and dementia Atherosclerosis
\end{abstract}

Other cardiovascular diseases

Hypertension

AGE-3 ${ }^{\mathrm{a}}$

MGO-derived AGEs

Osteoporosis, osteoarthritis, Undefined AGE and sarcopenia
AGE-2, AGE-3

CML, CEL, and argpyrimidine and other ligands for RAGE

AGE-1, MGO-derived AGEs

TAGEs and other AGE
Accelerates retinopathy by upregulating VEGF mRNA expression levels, and stimulating DNA synthesis and tube formation in microvascular endothelial cell through interacting with RAGEs Induces apoptotic cell death in human mesangial cells, and causes hyperfiltration and microalbuminuria by stimulating secretion of VEGF and monocyte chemoattractant protein-1

Associated with the development of large- and small-fibre dysfunction

Neurodegeneration associated with upregulation of RAGEs and the MAPK pathway; AGEs also induce protein aggregation and cross-linking between molecules, the formation of Lewy bodies and neuronal apoptosis

Stimulate the growth and migration of human melanoma cells

AGEs, via binding to RAGEs, result in sustained inflammation, which leads to metabolic reprogramming, and genomic instability, and may result in oncogenic transformation, telomere elongation and increased angiogenesis

May affect hippocampal neurons, and increase the percentage of apoptotic neurones in the hippocampus AGEs trigger inflammation and cell proliferation, contributing to the development of vascular dysfunction; glycation of apolipoprotein B100 increases the atherogenicity of low-density lipoproteins; AGEs influence platelet activation, thrombosis and hypercoagulability

Causes fibrosis, hypertrophy, oxidative stress and an exacerbated inflammatory response

Vascular endothelial damage by increasing oxidative stress, reduction of NO-dependent vasorelaxation; increasing arterial stiffness by cross-linking of extracellular matrix proteins

AGEs accumulate in bones, joints and skeletal muscles, impairing the biomechanical properties of the tissues; AGE-induced chronic inflammation may stimulate osteoblasts, osteoclasts and myocytes, resulting in degradation of bones, cartilage and muscles

${ }^{a}$ Based on the assumption that the AGE used from Sigma-Aldrich (Merck KGaA) is a product with the cat. no. 121800, as the authors did not specify. AGE, advanced glycation end product; RAGE, receptor for AGE; TAGE, toxic AGE; VEGF, vascular endothelial growth factor.

potent neurotoxic effects in cortical neuronal cells, and the effect was stronger than that of Glu-AGEs and CML. Additionally, another line of evidence which has shown that GA-AGE is involved in neurodegeneration, is that the neurotoxic effects of serum AGE fractions from diabetic nephropathy in haemodialysis patients were completely attenuated by the addition of an anti-GA-AGE antibody, but not by antibodies against other types AGEs (30).

On the basis of histological analysis and determination of serum AGE-2 levels, the relationship between the presence of this 
antigen and Alzheimer's disease has been suggested. AGE-2 is primarily detected in the cytosol of neurons of the hippocampus and para-hippocampal gyrus, but not in the senile plaques in the brains of AD patients, where CML was present (31).

Acetaldehyde, a product of ethanol metabolism, is a two-carbon carbonyl compound that can react with nucleophiles to form covalent addition products. It has been shown that AA-AGE may participate in the degeneration of neurons as the AA-AGE epitope has been detected in the brains of alcoholic individuals (12).

In the context of neurodegenerative diseases, the proper functioning of both neurons and the glial cells is of paramount importance. The effects of TAGEs (AGE-2 and AGE-3) on cultured Schwann cells has been assessed. Cell viability, proliferation and the production of proinflammatory cytokines were all substantially affected by treatment with TAGEs (12).

\section{TAGEs in cancer}

AGEs have been known to promote mutagenesis and stimulate proliferation and migration of cancer cells through activation of RAGEs. Stimulation of the receptors upon binding of its ligands, for example with interalia AGE, leads to the activation of several molecular signalling pathways, including the PI3K/AKT, JAK/STAT, NF-кB, Ras/MAPK, Rac1/cdc42, p44/p42, p38 and SAP/JNK/MAPK pathways, which contribute to the expression of transcription factors, such as $N F-\kappa B$, STAT3, HIF-1 $\alpha$, AP-1 and CREB. AGE-RAGE interactions also result in activation of NADPH oxidases, leading to increased intracellular oxidative stress. These inflammatory mediators induce epigenetic changes in pre-malignant lesions and silence tumour suppressor genes (17).

RAGE expression is upregulated in a vast majority of cancers, but its expression is very low under physiological conditions. Specific examples of types of cancer in which RAGE expression is upregulated include colorectal cancer (32), pancreatic cancer (33), prostate cancer (34), lung cancer (35) and breast cancer, and in breast cancer, it has been reported that the RAGE rs1800624 polymorphism may increase the risk of breast cancer (36).

It has been shown that TAGEs may contribute to the pathogenesis of neoplasia and cancer development. In human pancreatic cancer cells treated with 1-4 mmol/1 GA for $24 \mathrm{~h}$, the cell viability and intracellular levels of GA-AGEs, as well as heat shock proteins $90 \alpha, 90 \beta, 70,27$ were analyzed. Cell viability decreased to almost $0 \%$ when cells were treated with $4 \mathrm{mmol} / \mathrm{l} \mathrm{GA}$, and the levels of heat shock proteins increased significantly. It was concluded that intracellular GA-AGEs induce pancreatic cancer cell death. Additionally, their secretion may promote the proliferation of other cancer cells (37).

\section{Dietary AGEs (dAGEs)}

AGEs can also be a toxic component of food. It is estimated that an average human diet consists of $75 \mathrm{mg}$ AGEs per day (38). It is not clear how much dAGE is absorbed in the digestive system; one study has stated that $10-30 \%$ is absorbed (10), whereas another report has stated $30-80 \%$ is abosrbed (38). Absorbed glycation end-products are biotransformed and excreted, or otherwise accumulate in various tissues. The levels of accumulated AGEs in tissues can be estimated using the non-invasive skin autofluorescence measurement method (39).

The body's ability to detoxify AGE products is difficult to assess. Glycated proteins, particularly large ones, are resistant to proteolytic enzymes, and this makes it several times more difficult to eliminate them from the body. However, certain AGEs are bound to AGER1 receptors located on macrophages, T-lymphocytes, endothelial cells, mesangial cells, fibroblasts, smooth muscle cells and neuronal cells, which are then excreted by the kidney (10). It is estimated that $30 \%$ of dAGEs are removed in the urine, provided the patient has a healthy kidney, otherwise this percentage will be lower (40).

\section{TAGEs for diagnosis and treatment of diseases}

It has been suggested that the serum levels of toxic AGEs are correlated with the progression of degenerative diseases, such as atherosclerosis or diabetes. It was also found that higher levels of TAGEs are associated with overeating, a lack of exercise or excessive ingestion of sugars and dAGEs. It is therefore proposed that the serum levels of TAGEs may be a promising novel biomarker for the onset and progression of lifestyle-related diseases (41).

Sato et al (13) proposed TAGEs may serve as biomarkers for Alzheimer's disease, and suggested that assessing TAGE levels as a diagnostic tool may improve diagnosis and thus treatment of patients with this disorder.

There are also specific proposals to apply knowledge regarding AGEs and TAGEs for therapeutic purposes. It was suggested that inhibition of the interactions between a TAGE and its RAGE, using an anti-RAGE antibody, was a suitable candidate for treatment of patients with malignant melanoma (12). FPS-ZM1 or other specific antagonists for RAGEs may also be useful in inhibiting RAGEs, and may thus also serve as a potential therapeutic option for management of diabetic vascular complications (29).

Other approaches to reduce the detrimental effects of AGEs may be to inhibit their formation, or the use of compounds that can break the cross-linked bonds, such as aminoguanidine and pyridoxamine, phenylthiazole, 4,5-dimethyl-3-phenyl-acyl-thiazole chloride and ALT-711 (42). These are synthetic substances, and, unfortunately, no endogenous analogues have been identified as of yet. The downside of these compounds is that they may exert a certain degree of cytotoxicity, and this has complicated the development of a clinically suitable anti-AGE treatment. It has not been clearly established whether these compounds affects both TAGE and non-TAGE metabolism; however, it is worth exploring the therapeutic value of such strategies, and possibly applying them in clinical practice.

\section{Methodology for determining AGEs in biological materials}

Determination of AGEs in tissues is problematic. It is important to pay particular attention to the diversity of compounds that should be analysed $(4,11)$. Additionally, a multitude of conditions that can influence the glycation of AGEs (such as $\mathrm{pH}$, presence and the amount of free radicals and metal ions, amongst others), and the type and concentration of substrates 
need to be taken into consideration (4). Moreover, AGEs are typically present in small amounts in vivo $(14,43)$. Their isolation from tissues can cause undesirable chemical modifications and the formation of artefacts (44). Nevertheless, research on the content of AGEs in serum, in tissues and in food is very desirable, particularly for diagnostic and therapeutic purposes $(14,21,38,41,45,46)$.

A number of methods for determining AGEs in samples are available. Amongst the most frequently used are chromatographic methods coupled with mass spectroscopy $(11,14,44,46)$. Equally popular are immunoenzymatic methods using antibodies $(1,4,44,47)$; however, the specificity of the antibodies $(11,14)$ or the low quantities of antigens in the sample can cause difficulties (1). The natural properties of AGEs associated with fluorescence can also be exploited in fluorometric methods $(43,45,47)$; unfortunately, the fluorescence of other components in the sample often obfuscates the results (11). Thus, at present, there is no one specific and sensitive method to measure AGE content in samples (11).

\section{Summary and conclusions}

Despite the fact that methods for determination of the entire range of possible AGEs in biological samples is limited and challenging, it is still recommended to attempt measurement to reduce errors. Numerous studies have shown the association between AGEs and their negative effects, such as oxidative stress, inflammatory processes, and the formation of cross-links, which can alter the biochemical properties of proteins $(12,48)$.

AGEs can be classified as toxic or non-toxic AGEs, however it seems that the effect of each type of AGE may vary based on the specific conditions. A significant challenge in the study of AGEs is that the structures of AGE-1 through AGE-4 and AA-AGE are not defined. Additionally, the epitopes recognized by the antibodies in the study of TAGEs have not been determined. Another problem faced by researchers examining AGEs is the impact of albumin-AGE. The formation of AGE is complex, and is partially dependent on non-enzymatic glycation, and it is difficult to control the course of this process to obtain a homogeneous reaction product. The levels of AGEs or TAGEs in serum or tissues is also very small, hence the difficulty in accurately determining their concentration in biological samples. Thus, there is an urgent need to improve our understanding of the effects of glycated agents, as it may improve our understanding of diabetic complications, and other processes associated with oxidative stress caused by glycation.

It is also important to educate individuals on the risk of AGE toxicity, instilling the importance of a healthy diet, and encouraging them to choose food products that are not processed in high temperature conditions. It is also worth raising patients' awareness on preventing the effects of glycation, used to protect food supplies. Natural anti-glycation agents include anthocyanins and ellagic acid, which are derived from vegetables and fruits, and are also active ingredients contained in green tea; garlic; resveratrol; red wine; curcumin; cinnamic acid derivatives, such as ferulic acid; quercetin, which is found in several plants, caffeic acid from Ilex paraguariensis and numerous other food stuffs $(49,50)$. Lowering the intake of
dAGEs can reduce the risk of diabetes, cardiovascular complications and other glycation-related diseases.

Due to the ability of AGEs to increase the risk of diseases and promote neoplastic changes, AGEs are becoming an increasingly popular subject of study in the field of toxicology. However, it is extremely difficult to define the strict toxicological parameters of the various types of AGEs in the literature. At present the following parameters are used: No-Observed-Effect-Level, No-Observed-Adverse-Effect-Le vel, Permissible Exposure Limit and Time-Weighted Average. An increasing interest in the field of AGEs may emphasize the importance of the role of AGEs in the pathological mechanisms of various diseases, and improve public awarness of the risks associated with ingestion of food-derived AGEs.

\section{Acknowledgements}

I would like to thank Miss Karolina Nowakowska (Department of Internal Medicine, Pneumology and Allergology, Wroclaw Medical University, Poland) for preparing the figures and linguistic correction, and Dr Mariusz Bromke (Department of Medical Biochemistry, Wroclaw Medical University, Poland) for proofreading this review.

\section{Funding}

No funding was received.

\section{Availability of data and materials}

Not applicable.

\section{Authors' contributions}

AK wrote, edited and approved the final manuscript.

\section{Ethics approval and consent to participate}

Not applicable.

\section{Patient consent for publication}

Not applicable.

\section{Competing interests}

The authors declare that they have no competing interests.

\section{References}

1. Morioka Y, Teshigawara K, Tomono Y, Wang D, Izushi Y, Wake H, Liu K, Takahashi HK, Mori S and Nishibori M: The specific localization of advanced glycation end-products (AGEs) in rat pancreatic islets. J Pharmacol Sci 134: 218-224, 2017.

2. Miroliaei M, Aminjafari A, Ślusarczyk S, Nawrot-Hadzik I, Rahimmalek M and Matkowski A: Inhibition of glycation-induced cytotoxicity, protein glycation, and activity of proteolytic enzymes by extract from Perovskia atriplicifolia roots. Pharmacogn Mag 13 (Suppl 3): S676-S683, 2017.

3. Loske C, Neumann A, Cunningham AM, Nichol K, Schinzel R, Riederer P and Münch G: Cytotoxicity of advanced glycation endproducts is mediated by oxidative stress. J Neural Transm (Vienna) 105: 1005-1015, 1998 
4. Gkogkolou P and Böhm M: Advanced glycation end products: Keyplayers in skin aging? Dermatoendocrinol 4: 259-270, 2012.

5. Boyanova M and Huppertz B: Cytotoxic effect of advanced glycation end products. Biotechnol Biotechnol Equip 23: 1072-1078, 2009.

6. Wu B, Yu L, Hu P, Lu Y, Li J, Wei Y and He R: GRP78 protects $\mathrm{CHO}$ cells from ribosylation. Biochim Biophys Acta Mol Cell Res 1865: 629-637, 2018

7. Stinghen AE, Massy ZA, Vlassara H, Striker GE and Boullier A: Uremic toxicity of advanced glycation end products in CKD. J Am Soc Nephrol 27: 354-370, 2016.

8. Sergi D, Boulestin H, Campbell FM and Williams LM: The role of dietary advanced glycation end products in metabolic dysfunction. Mol Nutr Food Res 65: e1900934, 2021.

9. Ali Khan MW, Banga K and Ali W: Gluco-oxidation of proteins in etiology of diabetic retinopathy. In: Diabetic retinopathy. InTech, 2012

10. Chen JH, Lin X, Bu C and Zhang X: Role of advanced glycation end products in mobility and considerations in possible dietary and nutritional intervention strategies. Nutr Metab (Lond) 15: 72 , 2018.

11. Perrone A, Giovino A, Benny J and Martinelli F: Advanced glycation end products (AGEs): Biochemistry, signaling, analytical methods, and epigenetic effects. Oxid Med Cell Longev 2020: 3818196, 2020.

12. Takeuchi M and Yamagishi S: TAGE (toxic AGEs) hypothesis in various chronic diseases. Med Hypotheses 63: 449-452, 2004

13. Sato T, Shimogaito N, Wu X, Kikuchi S, Yamagishi S and Takeuchi M: Toxic advanced glycation end products (TAGE) theory in Alzheimer's disease. Am J Alzheimers Dis Other Demen 21: 197-208, 2006.

14. Ashraf JM, Ahmad S, Choi I, Ahmad N, Farhan M, Tatyana G and Shahab U: Recent advances in detection of AGEs: Immunochemical, bioanalytical and biochemical approaches IUBMB Life 67: 897-913, 2015.

15. Federico G, Gori M, Randazzo E and Vierucci F: Skin advanced glycation end-products evaluation in infants according to the type of feeding and mother's smoking habits. SAGE Open Med 4: 2050312116682126, 2016.

16. Sellier C, Boulanger E, Maladry F, Tessier FJ, Lorenzi R, Nevière R, Desreumaux $\mathrm{P}$, Beuscart JB, Puisieux $\mathrm{F}$ and Grossin N: Acrylamide induces accelerated endothelial aging in a human cell model. Food Chem Toxicol 83: 140-145, 2015.

17. Palanissami G and Paul SFD: RAGE and its ligands: Molecular interplay between glycation, inflammation, and hallmarks of cancer-a review. Horm Cancer 9: 295-325, 2018.

18. Schröter D and Höhn A: Role of advanced glycation end products in carcinogenesis and their therapeutic implications. Curr Pharm Des 24: 5245-5251, 2019

19. ALjahdali $\mathrm{N}$ and Carbonero $\mathrm{F}$ : Impact of maillard reaction products on nutrition and health: Current knowledge and need to understand their fate in the human digestive system. Crit Rev Food Sci Nutr 59: 474-487, 2019.

20. Cho HJ, Xie C and Cai H: AGE-induced neuronal cell death is enhanced in G2019S LRRK2 mutation with increased RAGE expression. Transl Neurodegener 7: 1, 2018.

21. Takeuchi M, Sakasai-Sakai A, Takata T, Ueda T, Takino J, Tsutsumi M, Hyogo $\mathrm{H}$ and Yamagishi S: Serum levels of toxic AGEs (TAGE) may be a promising novel biomarker in development and progression of NASH. Med Hypotheses 84: 490-493, 2015.

22. Takeuchi M and Yamagishi S: Involvement of Toxic AGEs (TAGE) in the pathogenesis of diabetic vascular complications and Alzheimer's disease. J Alzheimers Dis 16: 845-858, 2009.

23. Sakasai-Sakai A, Takata T, Takino J and Takeuchi M: Impact of intracellular glyceraldehyde-derived advanced glycation end-products on human hepatocyte cell death. Sci Rep 7: 14282, 2017.

24. Liu X, Zheng L, Zhang R, Liu G, Xiao S, Qiao X, Wu Y and Gong Z: Toxicological evaluation of advanced glycation end product $\mathrm{N} \varepsilon$-(carboxymethyl)lysine: Acute and subacute oral toxicity studies. Regul Toxicol Pharmacol 77: 65-74, 2016.

25. Chou SM, Wang HS, Taniguchi A and Bucala R: Advanced glycation endproducts in neurofilament conglomeration of motoneurons in familial and sporadic amyotrophic lateral sclerosis. Mol Med 4: 324-332, 1998.

26. Bassi AM, Ledda S, Valentini S, De Pascale MC, Rossi S, Odetti P and Cottalasso D: Damaging effects of advanced glycation end-products in the murine macrophage cell line J774A.1. Toxicol In Vitro 16: 339-347, 2002.
27. Chowdhury AA, Gawali NB, Bulani VD, Kothavade PS, Mestry SN, Deshpande PS and Juvekar AR: In vitro antiglycating effect and in vivo neuroprotective activity of Trigonelline in d-galactose induced cognitive impairment. Pharmacol Rep 70: 372-377, 2018.

28. Sampath C, Zhu Y, Sang S and Ahmedna M: Bioactive compounds isolated from apple, tea, and ginger protect against dicarbonyl induced stress in cultured human retinal epithelial cells. Phytomedicine 23: 200-213, 2016.

29. Kim JH, Kim KA, Shin YJ, Kim H, Majid A and Bae ON: Methylglyoxal induced advanced glycation end products (AGE)/receptor for AGE (RAGE)-mediated angiogenic impairment in bone marrow-derived endothelial progenitor cells. J Toxicol Environ Health A 81: 266-277, 2018.

30. Takeuchi M, Takino JI, Sakasai-Sakai A, Takata $\mathrm{T}$ and Tsutsumi M: Toxic AGE (TAGE) theory for the pathophysiology of the Onset/Progression of NAFLD and ALD. Nutrients 9: 634, 2017.

31. Takeuchi M, Sato T, Takino J, Kobayashi Y, Furuno S, Kikuchi S and Yamagishi S: Diagnostic utility of serum or cerebrospinal fluid levels of toxic advanced glycation end-products (TAGE) in early detection of Alzheimer's disease. Med Hypotheses 69: 1358-1366, 2007

32. Azizian-Farsani F, Abedpoor N, Hasan Sheikhha M, Gure AO, Nasr-Esfahani MH and Ghaedi K: Receptor for advanced glycation end products acts as a fuel to colorectal cancer development. Front Oncol 10: 552283, 2020.

33. Swami P, Thiyagarajan S, Vidger A, Indurthi VSK, Vetter SW and Leclerc E: Rage up-regulation differently affects cell proliferation and migration in pancreatic cancer cells. Int J Mol Sci 21 7723,2020

34. Akkus G, Izol V, Ok F, Evran M, Inceman M, Erdogan S, Kaplan HM, Sert M and Tetiker T: Possible role of the receptor of advanced glycation end products (RAGE) in the clinical course of prostate neoplasia in patients with and without type 2 diabetes mellitus. Int J Clin Pract: Sep 21, 2020 (Epub ahead of print). doi: 10.1111/ijcp.13723.

35. Chen MC, Chen KC, Chang GC, Lin H, Wu CC, Kao WH, Teng CJ, Hsu SL and Yang TY: RAGE acts as an oncogenic role and promotes the metastasis of human lung cancer. Cell Death Dis 11: 265, 2020.

36. Zhang W, Deng X, Tang R and Wang H: Receptor for advanced glycation end-product rs1800624 polymorphism contributes to increase breast cancer risk: Evidence from a meta-analysis. Medicine (Baltimore) 99: e22775, 2020.

37. Takata T, Ueda T, Sakasai-Sakai A and Takeuchi M: Generation of glyceraldehyde-derived advanced glycation end-products in pancreatic cancer cells and the potential of tumor promotion. World J Gastroenterol 23: 4910-4919, 2017.

38. Waqas K, Chen J, van der Eerden BCJ, Ikram MA, Uitterlinden AG, Voortman T and Zillikens M: Dietary advanced glycation end-products (dAGEs) intake and bone health: A cross-sectional analysis in the rotterdam study. Nutrients 12: 2377, 2020.

39. Yamagishi $\mathrm{S}$ and Matsui $\mathrm{T}$ : Role of ligands of receptor for advanced glycation end products (RAGE) in peripheral artery disease. Rejuvenation Res 21: 456-463, 2018.

40. Tessier FJ, Boulanger E and Howsam M: Metabolic transit of dietary advanced glycation end-products-the case of $\mathrm{N}^{\varepsilon}$-carboxymethyllysine. Glycoconj J: Sep 29, 2020 (Epub ahead of print). doi: 10.1007/s10719-020-09950-y.

41. Takeuchi M: Serum levels of toxic AGEs (TAGE) may be a promising novel biomarker for the onset/progression of lifestyle-related diseases. Diagnostics (Basel) 6: 23, 2016.

42. Kuzan A, Chwiłkowska A, Kobielarz M, Pezowicz C and Gamian A: Glycation of extracellular matrix proteins and its role in atherosclerosis. Postepy Hig Med Dosw (Online) 66: 804-809, 2012.

43. Kuzan A, Chwiłkowska A, Maksymowicz K, BronowickaSzydełko A, Stach K, Pezowicz C and Gamian A: Advanced glycation end products as a source of artifacts in immunoenzymatic methods. Glycoconj J 35: 95-103, 2018

44. Humeny A, Kislinger T, Becker CM and Pischetsrieder M: Qualitative determination of specific protein glycation products by matrix-assisted laser desorption/ionization mass spectrometry Peptide mapping. J Agric Food Chem 50: 2153-2160, 2002.

45. Yamagishi S and Matsui T: Role of Hyperglycemia-induced advanced glycation end product (AGE) accumulation in atherosclerosis. Ann Vasc Dis 11: 253-258, 2018. 
46. Kim Y, Keogh JB, Deo P and Clifton PM: Differential effects of dietary patterns on advanced glycation end products: A randomized crossover study. Nutrients 12: 1767, 2020.

47. Münch G, Keis R, Wessels A, Riederer P, Bahner U, Heidland A Niwa T, Lemke HD and Schinzel R: Determination of advanced glycation end products in serum by fluorescence spectroscopy and competitive ELISA. Eur J Clin Chem Clin Biochem 35: 669-677, 1997.

48. Kuzan A, Michel O and Gamian A: Glycation of matrix proteins in the artery inhibits migration of smooth muscle cells from the media to the intima. Folia Biol (Praha) 63: 105-114, 2017.

49. Younus H and Anwar S: Prevention of Non-enzymatic glycosylation (Glycation): Implication in the treatment of diabetic complication. Int J Health Sci (Qassim) 10: 261-277, 2016.

50. Hashemzaei M, Tabrizian K, Alizadeh Z, Pasandideh S, Rezaee R, Mamoulakis C, Tsatsakis A, Skaperda Z, Kouretas D and Shahraki J: Resveratrol, curcumin and gallic acid attenuate glyoxal-induced damage to rat renal cells. Toxicol Rep 7: $1571-1577,2020$

51. Schalkwijk CG and Stehouwer CDA: Methylglyoxal, a highly reactive dicarbonyl compound, in diabetes, its vascular complications and other age-related diseases. Physiol Rev 100: 407-461, 2020 .

52. Byun K, Bayarsaikhan D, Bayarsaikhan E, Son M, Oh S, Lee J, Son HI, Won MH, Kim SU, Song BJ and Lee B: Microglial AGE-albumin is critical in promoting alcohol-induced neurodegeneration in rats and humans. PLoS One 9: e104699, 2014.

53. Bayarsaikhan E, Bayarsaikhan D, Lee J, Son M, Oh S, Moon J, Park HJ, Roshini A, Kim SU, Song BJ, et al: Microglial AGE-albumin is critical for neuronal death in Parkinson's disease: A possible implication for theranostics. Int J Nanomedicine 10: 281-292, 2016.
54. Fleitas C, Piñol-Ripoll G, Marfull P, Rocandio D, Ferrer I, Rampon C, Egea $\mathrm{J}$ and Espinet $\mathrm{C}$ : proBDNF is modified by advanced glycation end products in Alzheimer's disease and causes neuronal apoptosis by inducing p75 neurotrophin receptor processing. Mol Brain 11: 68, 2018.

55. Moldogazieva NT, Mokhosoev IM, Mel'nikova TI, Porozov YB and Terentiev AA: Oxidative stress and advanced lipoxidation and glycation end products (ALEs and AGEs) in aging and age-related diseases. Oxid Med Cell Longev 2019: 3085756, 2019.

56. Wang X, Chen XX, Yu HT, Tan Y, Lin Q, Keller BB, Zheng Y and Cai L: Engineered cardiac tissues: A novel in vitro model to investigate the pathophysiology of mouse diabetic cardiomyopathy. Acta Pharmacol Sin: Oct 9, 2020 (Epub ahead of print). doi: 10.1038/s41401-020-00538-8.

57. Wang WC, Lee JA and Chou CK: Open access short review evolving evidence of methylglyoxal and dicarbonyl stress related diseases from diabetic to non-diabetic models. Pharm Anal Acta 7: 4, 2016

58. Takata T, Sakasai-Sakai A and Takeuchi M: Impact of intracellular toxic advanced glycation end-products (TAGE) on murine myoblast cell death. Diabetol Metab Syndr 12: 54, 2020.

This work is licensed under a Creative Commons Attribution-NonCommercial-NoDerivatives 4.0 International (CC BY-NC-ND 4.0) License. 